\title{
H $\alpha$ Emission in the Giant Components of RS CVn Systems
}

\author{
A. Frasca, S. Catalano \\ Istituto di Astronomia, Università di Catania \\ viale A. Doria 6, I-95125 Catania, Italy
}

\section{Introduction}

The $\mathrm{H} \alpha$ line of hydrogen is a very powerful tool for the study of the solar chromosphere (plages, flares, prominences). Although it is difficult to derive in a simple way quantitative information, like chromospheric temperature and electron density, because of the very extensive depth of its formation and the very complex mechanism of excitation, $\mathrm{H} \alpha$ has been successfully used to study the chromospheres of active stars (Herbig, 1985). Moreover this diagnostic has proved to be very useful for the study of surface inhomogeneities and transient phenomena like flares in systems of the RS CVn type and other active stars (Bopp, 1981; Bopp et al., 1988; Fraquelli, 1982).

With the aim of investigating the behaviour of chromospheric activity, we have undertaken an extensive monitoring of $\mathrm{H} \alpha$ emission in a selected group of $\mathrm{RS}$ CVn binaries (Strassmeier et al., 1988). We report here preliminary results of the observations obtained in 1988-1989.

\section{Observations and reduction}

All the spectra here discussed were acquired with a CCD camera at the Echelle spectrograph of the $91 \mathrm{~cm}$ telescope of Catania Observatory. We used the spectrograph in a low-dispersion configuration which gives a spectral resolution of about $0.9 \AA /$ pixel. Exposure times were chosen to obtain spectra with a $\mathrm{S} / \mathrm{N}$ ratio of 100-150. The spectrum extraction from the CCD images was performed following a standard procedure (subtraction of the bias level, division by a continuous spectrum given by a halogenic lamp etc.). Repeated spectra of a standard star allowed us to verify that an accuracy of about $1 \%$ was reached in each point of the well exposed spectra, as expected for a ratio $\mathrm{S} / \mathrm{N} \sim 100$.

The net chromospheric $\mathrm{H} \alpha$ emission of the binary systems was defined as the difference of the observed spectrum and a "composite" spectrum made up of the weighted sum of spectra of non active stars similar in spectral type to the primary 
and secondary star of each system. For each spectrum we have shifted in wavelength the two components of this composite spectrum to compensate for the radial velocity changes due to the orbital motion. We have also taken into account the rotational broadening of the reference star $\mathrm{H} \alpha$ profiles every time this was larger than the spectral resolution. This method enable us to identify the component in the binary responsible for the $\mathrm{H} \alpha$ emission.

\section{Results}

Time variability of the net $\mathrm{H} \alpha$ emission modulated with the orbital period was detected in nearly all of the 10 systems for which repeated observations were obtained. Equivalent width variations ranged from $0.1 \AA$ to $1.3 \AA$ (Table).

Table: Net $\mathrm{H} \alpha$ equivalent width variations

\begin{tabular}{lccc}
\hline Star & $P_{\text {orb }}($ days $)$ & Spectral types (Hot/Cool $)$ & $\Delta W_{H \alpha}(\AA)$ \\
\hline RS CVn & 4.80 & F5 IV / K0 IV & 0.4 \\
HR 5110 & 2.61 & F2 IV / K2 IV & 0.3 \\
HR 5321 & 605.8 & K0 III & 0.1 \\
HD 163621 & $?$ & F / G5 IV & 0.2 \\
Z Her & 3.99 & F4 V-IV / K0 IV & 0.2 \\
AR Lac & 1.98 & G2 IV / K0 IV & 0.4 \\
RT Lac & 5.07 & G9 IV / K1 IV & 1.1 \\
HK Lac & 24.42 & F1 IV / K0 III & 2.3 \\
SZ Psc & 3.97 & F8 IV / K1 IV & 1.3 \\
UX Ari & 6.44 & G5 V / K0 IV & 0.7 \\
HR 1099 & 2.84 & G5 IV / K1 IV & 1.1 \\
\hline
\end{tabular}

The variation with the orbital phase of the net $\mathrm{H} \alpha$ emission equivalent width for HR 1099 is reported in Fig. 1, as example of the present results. For systems like $\mathrm{ZHer}$ or HR 5110 such variations do not seem to be correlated with the orbital phase. Although no significant variation (above the $2 \%$ level) were found with time scales of a few hours, random or flare-like variations with time scales of days were detected for many systems. In particular we observed a giant $\mathrm{H} \alpha$ flare on HK Lac (a system with an orbital period of about 24 days), which showed an emission enhancement by a factor of $\sim 2$ and lasted about 6 days (Fig. 2). The flare peak flux at the stellar surface has been estimated to be $\sim 3 \times 10^{7} \mathrm{erg} \mathrm{cm}^{-2} \mathrm{~s}^{-1}$ and the total energy released was about $10^{38} \mathrm{erg}$. This seems to be one of the most powerful flares observed until now in systems of this kind (Catalano, 1990).

Acknowledgements: This work was supported by the MURST through the University of Catania, the Osservatorio Astrofisico di Catania and the GNA (CNR). The extensive use of the computer facilities of the Catania ASTRONET Site is also acknowledged. 


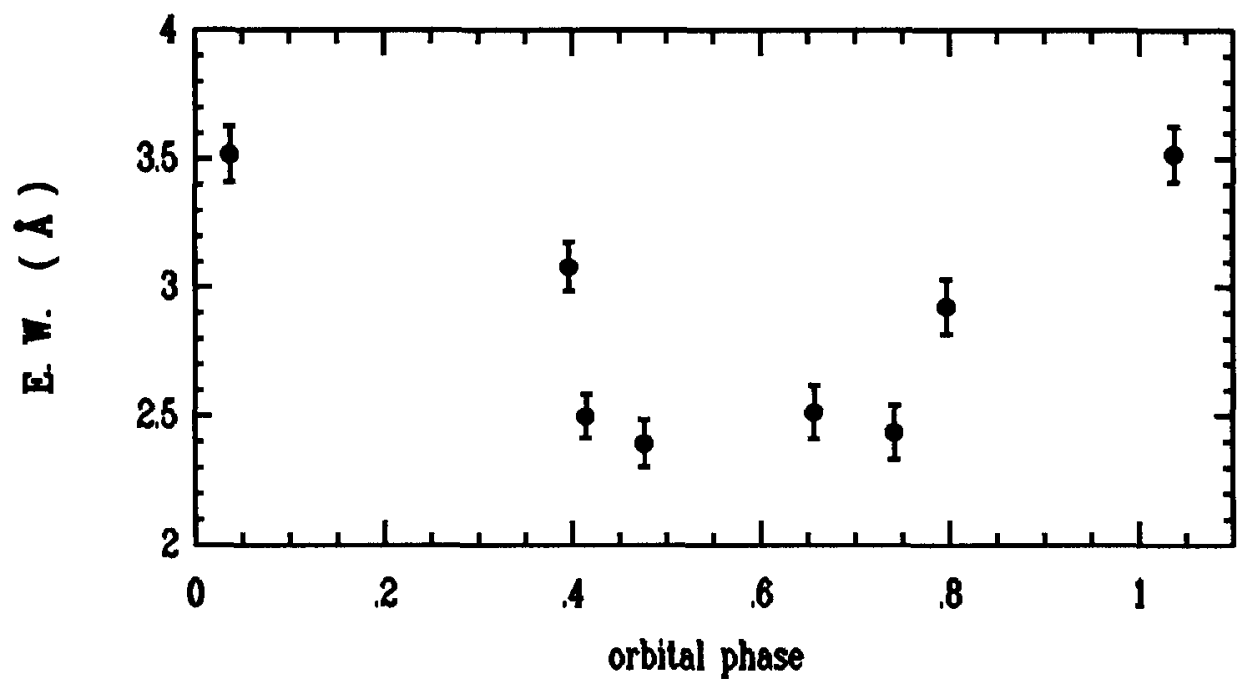

Fig. 1. Net $\mathrm{H} \alpha$ equivalent width of HR 1099 plotted against orbital phase.

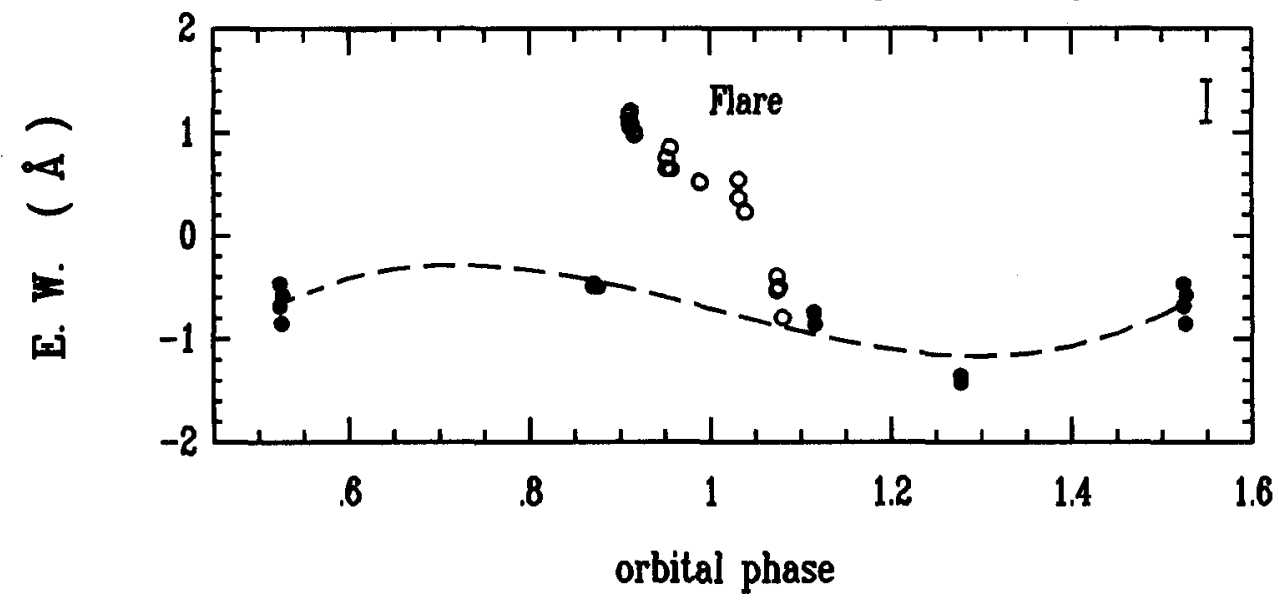

Fig. 2. Observed $\mathrm{H} \alpha$ emission of HK Lac versus orbital phase. The open circles relate to the flare-like event observed in 1989 between 25 and 29 of September.

\section{References}

Bopp, B.W.: 1981, Astron. J. 86, 771

Bopp, B.W., Dempsey, R.C., Maniak, S.: 1988, Astrophys. J. Suppl. Ser. 68, 803

Catalano, S.: 1990, in Active Close Binaries, eds. C. Ibanoglu and I. Yavuz, in press

Fraquelli, D.A.: 1982, Astrophys. J. Lett. 254, L41

Herbig, G. H.: 1985, Astrophys. J. 289, 269

Strassmeier, K.G., Hall, D.S., Zeilik, M., Nelson, E., Eker, Z., Fekel, F.C.: 1987, Astron. Astrophys. Suppl. Ser. 72, 291 